\title{
Minimax Estimation of the Scale Parameter of Laplace Distribution under Modified Linear Exponential (MLINEX) Loss Function
}

\author{
M. R. Hasan* \\ Department of Business Administration, Leading University, Sylhet, Bangladesh \\ Received 23 January 2019, accepted in final revised form 27 May 2019
}

\begin{abstract}
The main objective of this paper is to find the minimax estimator of the scale parameter of Laplace distribution under MLINEX loss function by applying the theorem of Lehmann (1950). The estimator is then compared with classical estimator like moment estimator with respect to mean square errors (MSEs) through R- Code simulation. The result has shown that the minimax estimator under MLINEX loss function is better than moment estimator for all sample sizes. Finally, mean square errors of different estimators corresponding to sample size are presented graphically.
\end{abstract}

Keywords: Minimax estimator; Moment estimator; Jeffrey prior; Bayes estimator; MLINEX Loss Function.

(c) 2019 JSR Publications. ISSN: 2070-0237 (Print); 2070-0245 (Online). All rights reserved.

doi: http://dx.doi.org/10.3329/jsr.v11i3.39953 J. Sci. Res. 11 (3), 273-284 (2019)

\section{Introduction}

Minimax estimation is a non- classical estimation approach in statistical inference, which was introduced by Abraham Wald [1] from the concept of Game theory. This estimation theory has drawn great attention from many researchers. Jasim Al-taie [2] derived the minimax estimators of the scale parameter of Gamma distribution where the shape parameter is known, under quadratic and Modified LINEX loss functions using Lehmann theorem (1950). Rasheed and AL-Shareefi [3] studied minimax estimation of the scale parameter of Laplace distribution under squared-log error loss function. Li [4] discussed minimax estimation of the parameter of Maxwell distribution under different loss functions. In another paper the same author [5] also estimated the shape parameter of Laplace distribution using Bayesian technique under a new loss function, which is a compound function of LINEX function. Hasan and Baizid [6] studied Bayesian estimation under different loss functions using gamma prior for the case of exponential distribution and compared among them as well as with the classical estimator named maximum likelihood estimator (MLE). Mahanta and Talukdar [7] estimated the parameter of

\footnotetext{
Corresponding author: rashidulhasan067@gmail.com
} 
Rayleigh distribution by adopting Bayesian approach under squared error, LINEX, MLINEX loss function. The performances of the obtained estimators for different types of loss functions were then compared. Laplace distribution is a very popular continuous probability distribution. It has generally two parameters. One is location parameter $\theta$ and other is scale parameter $\lambda$. Practically location parameter has limited use. Here only scale parameter is considered to estimate. A continuous random variable $X$ is said to have Laplace $(\theta, \lambda)$ distribution if its probability density function (pdf) is given by [8]

$$
f(x ; \lambda, \theta)= \begin{cases}\frac{1}{2 \lambda} \mathrm{e}^{-\frac{|\mathrm{x}-\theta|}{\lambda}} \quad ;-\infty<x<\infty,-\infty<\theta<\infty, \lambda>0 \\ 0 \quad ; \text { otherwise }\end{cases}
$$

Where, $\theta$ is the location parameter and $\lambda$ is the scale parameter.

The cumulative distribution function (cdf) of Laplace distribution is given by

$$
F(x ; \lambda, \theta)=\left\{\begin{array}{l}
1-\frac{1}{2} \exp \left(-\frac{x-\theta}{\lambda}\right) ; \text { if } \mathrm{x} \geq \theta \\
\frac{1}{2} \exp \left(\frac{\mathrm{x}-\theta}{\lambda}\right) ; \text { if } \mathrm{x}<\theta
\end{array}\right.
$$

Laplace distribution is used in hydrology to extreme events such as annual maximum oneday rainfall and river discharges. This distribution has also been used in speech recognition to model priors on discrete Furrier transform (DFT) coefficients and in joint photographic experts group (JPEG) image compression to model AC coefficients generated by a discrete cosine transform (DCT) [9].

\section{Prior and Posterior Density Function of Parameter $\lambda$}

Let us assume $\lambda$ has Jeffery prior information [10] [11] defined as $g(\lambda) \propto \sqrt{I(\lambda)}$. Where $I(\lambda)$ represents Fisher information which is defined as $I(\lambda)=-n E\left[\frac{\partial^{2} \ln f(x ; \lambda, \theta)}{\partial \lambda^{2}}\right]$ Here, $g(\lambda)=k \sqrt{I(\lambda)}=k \sqrt{-n E\left[\frac{\partial^{2} \ln f(x ; \lambda, \theta)}{\partial \lambda^{2}}\right]}$

Taking natural logarithm of (1) we obtain

$$
\begin{aligned}
& \ln f(x ; \lambda, \theta)=-\ln (2)-\ln (\lambda)-\frac{|x-\theta|}{\lambda} \Rightarrow \frac{\partial \ln f(x ; \lambda, \theta)}{\partial \lambda}=0-\frac{1}{\lambda}+\frac{|x-\theta|}{\lambda^{2}} \\
& \Rightarrow \frac{\partial^{2} \ln f(x ; \lambda, \theta)}{\partial \lambda^{2}}=\frac{1}{\lambda^{2}}-\frac{2|x-\theta|}{\lambda^{3}} \Rightarrow E\left[\frac{\partial^{2} \ln f(x ; \lambda, \theta)}{\partial \lambda^{2}}\right]=\frac{1}{\lambda^{2}}-\frac{2}{\lambda^{3}} E[|x-\theta|]
\end{aligned}
$$


Let, $X \sim$ Laplace $(\theta, \lambda)$ then, [12] $\left|x_{i}-\theta\right|=\lambda W$; Where, $W$ is standard exponential with mean and variance equal to one. Hence, $\quad\left|x_{i}-\theta\right| \sim \operatorname{Gamma}\left(1, \lambda^{-1}\right)$ Then, $E\left[\frac{\partial^{2} \ln f(x ; \lambda, \theta)}{\partial \lambda^{2}}\right]=\frac{1}{\lambda^{2}}-\frac{2}{\lambda^{3}} \cdot \lambda=-\frac{1}{\lambda^{2}}$

So, $g(\lambda)=k \sqrt{-n\left(-\frac{1}{\lambda^{2}}\right)}=\frac{1}{\lambda} k \sqrt{n}$

Now, the posterior density function of parameter $\lambda$ with Jeffrey prior is given by $h(\lambda / x)=\frac{g(\lambda) L\left(\lambda ; x_{1}, x_{2}, \ldots, x_{n}\right)}{\int_{0}^{\infty} g(\lambda) L\left(x_{1}, x_{2}, \ldots, x_{n}\right) d \lambda}=\frac{\frac{1}{\lambda} k \sqrt{n}\left(\frac{1}{2 \lambda}\right)^{n} e^{-\frac{\sum_{i=1}^{n}\left|x_{i}-\theta\right|}{\lambda}}}{\int_{0}^{\infty} \frac{1}{\lambda} k \sqrt{n}\left(\frac{1}{2 \lambda}\right)^{n} e^{\sum_{-\frac{i=1}{n}\left|x_{i}-\theta\right|}^{\lambda}} d \lambda}=\frac{e^{-\frac{\sum_{i=1}^{n}\left|x_{i}-\theta\right|}{\lambda} \lambda^{-n-1}}}{\int_{0}^{\infty} e^{-\frac{\sum_{i=1}^{n}\left|x_{i}-\theta\right|}{\lambda}} \lambda^{-n-1} d \lambda}$ $=\frac{e^{-\frac{\sum_{i=1}^{n}\left|x_{i}-\theta\right|}{\lambda} \lambda^{-n-1}}}{\left(\sum_{i=1}^{n}\left|x_{i}-\theta\right|\right)^{n}}=\frac{e^{-\frac{\sum_{i=1}^{n}\left|x_{i}-\theta\right|}{\lambda}}\left(\sum_{i=1}^{n}\left|x_{i}-\theta\right|\right)^{n}}{\lambda^{n+1} \Gamma n}=\frac{e^{-\frac{T}{\lambda}} T^{n}}{\lambda^{n+1} \Gamma n}$

So, $h(\lambda / x)=\frac{e^{-\frac{T}{\lambda}} T^{n}}{\lambda^{n+1} \Gamma n} ;$ Here, $T=\sum_{i=1}^{n}\left|x_{i}-\theta\right|$ follows $G\left(n, \lambda^{-1}\right)$

Therefore, the posterior density is recognized as the density of the Inverse Gamma (IG) distribution: $h(\lambda / x) \sim I G(n, T)$

\section{Different Estimators of Parameter $\lambda$}

Here, Bayes estimator of parameter $\lambda$ for MLINEX loss function along with moment estimator has been determined.

\subsection{Method of moment estimator (MME) of parameter $\lambda[13]$}

Let, $X=\left(X_{1}, X_{2}, \ldots \ldots, X_{n}\right)$ is a random sample of size $n$ drawn from density (1) with $E(X)=\theta$ and $\operatorname{Var}(X)=2 \lambda^{2}$. Then the sample mean $\bar{x}=\frac{1}{n} \sum_{i=1}^{n} x_{i}$ and the sample variance $S_{x}=\frac{1}{n} \sum_{i=1}^{n}\left(x_{i}-\bar{x}\right)^{2}$. Here, the method of moment estimator for $\theta$ is $\bar{x}=\frac{1}{n} \sum_{i=1}^{n} x_{i}$ and the method of moment estimator for $\lambda$ is $2 \lambda^{2}=S_{x}=\frac{1}{n} \sum_{i=1}^{n}\left(x_{i}-\bar{x}\right)^{2}$. Hence, $\hat{\lambda}_{M M E}=\sqrt{\frac{\sum_{i=1}^{n}\left(x_{i}-\bar{x}\right)^{2}}{2 n}}$ is the method of moment estimator (MME) of parameter $\lambda$. 


\subsection{Bayes estimator of parameter $\lambda$ for MLINEX loss function}

Let, the MLINEX loss function [14] is defined as

$$
L(\hat{\lambda}, \lambda)=\omega\left[\left(\frac{\hat{\lambda}}{\lambda}\right)^{c}-c \log \left(\frac{\hat{\lambda}}{\lambda}\right)-1\right] ; \omega>0, c \neq 0
$$

Now the risk function under MLINEX loss function is $R_{\hat{\lambda}_{B M L}}(\lambda)=E\left[L\left(\hat{\lambda}_{B M L}, \lambda\right)\right]$ For MLINEX loss function the Bayes estimator of parameter $\lambda$ is obtained by $\hat{\lambda}_{B M L}=\frac{1}{\left[E\left(\frac{1}{\lambda^{c}} / x\right)\right]^{\frac{1}{c}}}$

$$
\begin{aligned}
& \text { Now, } E\left(\frac{1}{\lambda^{c}} / x\right)=\int_{0}^{\infty} \frac{1}{\lambda^{c}} h(\lambda / x) d \lambda=\int_{0}^{\infty} \frac{1}{\lambda^{c}} \frac{e^{-\frac{\sum_{i=1}^{n}\left|x_{i}-\theta\right|}{\lambda}}\left(\sum_{i=1}^{n}\left|x_{i}-\theta\right|\right)^{n}}{\lambda^{n+1} \Gamma n} d \lambda \\
& =\frac{\left(\sum_{i=1}^{n}\left|x_{i}-\theta\right|\right)^{n}}{\Gamma n} \int_{0}^{\infty} e^{-\frac{\sum_{i=1}^{n}\left|x_{i}-\theta\right|}{\lambda}} \lambda^{-(n+c)-1} d \lambda=\frac{\left(\sum_{i=1}^{n}\left|x_{i}-\theta\right|\right)^{n}}{\Gamma n} \frac{\Gamma(n+c)}{\left(\sum_{i=1}^{n}\left|x_{i}-\theta\right|\right)^{n+c}}=\frac{\Gamma(n+c)}{\Gamma n\left(\sum_{i=1}^{n}\left|x_{i}-\theta\right|\right)^{c}}
\end{aligned}
$$

Therefore from (3) we get, $\hat{\lambda}_{B M L}=\left[\frac{\Gamma n}{\Gamma(n+c)}\right]^{\frac{1}{c}}\left(\sum_{i=1}^{n}\left|x_{i}-\theta\right|\right)$

Hence, $\hat{\lambda}_{B M L}=\left[\frac{\Gamma n}{\Gamma(n+c)}\right]^{\frac{1}{c}}\left(\sum_{i=1}^{n}\left|x_{i}-\theta\right|\right)$ is the Bayes estimator of $\lambda$ under MLINEX loss function.

\section{Minimax Estimator}

The derivation of minimax estimator depends primarily on a theorem due to Lehmann which can be stated as follows:

\subsection{Lehmann's Theorem [15]}

Let $\tau=\left\{F_{\theta}: \theta \in \Omega\right\}$ be a family of distribution function and $D$ be a class of estimators of $\theta$. Suppose, that $d^{*} \in D$ is a Bayes estimator against a prior distribution $\xi^{*}(\theta)$ on the parameter space $\Omega$, and the risk function $R\left(d^{*}, \theta\right)=$ constant on $\Omega$, then $d^{*}$ is a minimax estimator of $\theta$. The main results are contained in the following theorem. 


\subsection{Definition [16]}

An estimator $t^{*}$ is defined to be a minimax estimator if and only if: ${ }_{\theta}^{\operatorname{Sup}} R_{t^{*}}(\theta) \leq{ }_{\theta}^{\operatorname{Sup}} R_{t}(\theta)$ for every estimator $t$.

\subsection{Theorem}

Let, $X=\left(X_{1}, X_{2}, \ldots \ldots, X_{n}\right)$ be $n$ independent and identically distributed (iid) random variable drawn from the density function (1), then $\hat{\lambda}_{M E M L}=\left[\frac{\Gamma n}{\Gamma(n+c)}\right]^{\frac{1}{c}}\left(\sum_{i=1}^{n}\left|x_{i}-\theta\right|\right)$ is the minimax estimator of parameter $\lambda$ under MLINEX loss function.

To prove the theorem 4.3 we will use Lehmann's theorem, by showing that, the risk function of $\hat{\lambda}$ is a constant. Now we have to prove the theorem 4.3.

The risk function of the estimator $\hat{\lambda}_{B M L}$ is: $R_{\hat{\lambda}_{B M L}}(\lambda)=E\left[L\left(\hat{\lambda}_{B M L}, \lambda\right)\right]$ $=\omega\left[\frac{1}{\lambda^{c}} E\left(\hat{\lambda}_{B M L}^{c}\right)-c E\left\{\log \left(\hat{\lambda}_{B M L}\right)\right\}+c \log \lambda-1\right]$

Now, $E\left(\hat{\lambda}_{B M L}^{c}\right)=\frac{\Gamma n}{\Gamma(n+c)} E\left(T^{c}\right)=\frac{\Gamma n}{\Gamma(n+c)} \int_{0}^{\infty} t^{c} g(t) d t=\frac{\Gamma n}{\Gamma(n+c)} \int_{0}^{\infty} t^{c} \frac{1}{\Gamma n \lambda^{n}} e^{-\frac{t}{\lambda}} t^{n-1} d t$

$\Rightarrow E\left(\hat{\lambda}_{B M L}^{c}\right)=\frac{\Gamma n}{\Gamma(n+c)} \frac{\Gamma(n+c)}{\Gamma n} \lambda^{c}=\lambda^{c}$

Again, $E\left\{\log \left(\hat{\lambda}_{B M L}\right)\right\}=\log \left[\frac{\Gamma n}{\Gamma(n+c)}\right]^{\frac{1}{c}}+E\left\{\log \left(\sum_{i=1}^{n}\left|x_{i}-\theta\right|\right)\right\}$

$=\frac{1}{c} \log \left[\frac{\Gamma n}{\Gamma(n+c)}\right]+E\{\log (T)\}$

Now, $\mathrm{E}\{\log (\mathrm{T})\}=\int_{0}^{\infty} \log (t) g(t) d t=\int_{0}^{\infty} \log (t) \frac{1}{\Gamma n \lambda^{n}} e^{-\frac{t}{\lambda}} t^{n-1} d t$

Let, $y=\frac{t}{\lambda} \Rightarrow t=\lambda y$ So, $\frac{d t}{d y}=\lambda \Rightarrow d t=\lambda d y$

Then, $E\{\log (T)\}=\frac{1}{\Gamma n \lambda^{n}} \int_{0}^{\infty} \log (\lambda y)(\lambda y)^{n-1} e^{-y} \lambda d y=\frac{\log (\lambda)}{\Gamma n} \int_{0}^{\infty} y^{n-1} e^{-y} d y+\frac{1}{\Gamma n} \int_{0}^{\infty} \log (y) y^{n-1} e^{-y} d y$

So, $E\{\log (T)\}=\log (\lambda)+\frac{\Gamma^{\prime} n}{\Gamma n}$;

Where, $\Gamma^{\prime} n=\int_{0}^{\infty} \log (y) y^{n-1} e^{-y} d y$ is the first derivative of $\Gamma n$ with respect to $n$.

Now substituting the value of $E\{\log (T)\}$ in (6) we obtain 
$E\left\{\log \left(\hat{\lambda}_{B M L}\right)\right\}=\frac{1}{c} \log \left[\frac{\Gamma n}{\Gamma(n+c)}\right]+\log (\lambda)+\frac{\Gamma^{\prime} n}{\Gamma n}$

Again substituting (5) and (7) in (4) we obtain

$R_{\hat{\lambda}_{\text {BML }}}(\lambda)=\omega\left[\frac{1}{\lambda^{c}} \lambda^{c}-c \frac{1}{c} \log \left[\frac{\Gamma n}{\Gamma(n+c)}\right]-c \log (\lambda)-c \frac{\Gamma^{\prime} n}{\Gamma n}+c \log (\lambda)-1\right]$

$=\omega\left[1-\log \left[\frac{\Gamma n}{\Gamma(n+c)}\right]-c \frac{\Gamma^{\prime} n}{\Gamma n}-1\right]=-\omega\left[\log \left[\frac{\Gamma n}{\Gamma(n+c)}\right]+c \frac{\Gamma^{\prime} n}{\Gamma n}\right] ;$ Which is constant

So, according to the Lehmann's theorem it follows that, $\hat{\lambda}_{M E M L}=\left[\frac{\Gamma n}{\Gamma(n+c)}\right]^{\frac{1}{c}}\left(\sum_{i=1}^{n}\left|x_{i}-\theta\right|\right)$ is the minimax estimator of the parameter $\lambda$ of Laplace distribution under MLINEX loss function.

\section{Simulation Study}

In our simulation study $S=6000$ samples of size $n=5,10,20,30,50$ and 100 have been generated from Laplace distribution to represent small, moderate and large sample with the scale parameter $\lambda=0.5,1,1.5$ and 2 . To obtain the variance of $\hat{\lambda}$, we have used the true (assume) value of the parameter $\lambda$ under consideration. Again we have obtained the estimated value, bias and MSE of the estimators by using R- Code from the Laplace distribution. The results and their graphs using Minitab- 18 have presented in the following Tables (1-4) and Figs (1-4) respectively. In order to compare estimators, $\hat{\lambda}_{M M E}$ and $\hat{\lambda}_{M E M L}$ we have considered MSE of the estimators. The MSE of the estimator $\hat{\lambda}$ is defined as: $\operatorname{MSE}(\hat{\lambda})=E[\hat{\lambda}-\lambda]^{2}=\operatorname{Var}(\hat{\lambda})+[\operatorname{Bias}(\hat{\lambda})]^{2}$

Table 1. Estimated value, Bias and MSE of different estimators of parameter $\lambda$ of Laplace distribution when $\lambda=0.5, \theta=1$ and $\mathrm{c}=0.5$

\begin{tabular}{llll}
\hline $\begin{array}{l}\text { Sample } \\
\text { Size }(\mathrm{n})\end{array}$ & Criteria & $\hat{\lambda}_{M M E}$ & $\hat{\lambda_{M E M L}}$ \\
\hline 5 & Estimated Value & 0.1408 & 0.1488 \\
& Bias & -0.2289 & -0.1321 \\
& MSE & 0.0769 & 0.0513 \\
10 & Estimated Value & 0.3717 & 0.3706 \\
& Bias & -0.1912 & -0.1432 \\
20 & MSE & 0.0528 & 0.0373 \\
& Estimated Value & 0.1718 & 0.3350 \\
& Bias & -0.1703 & -0.1514 \\
30 & MSE & 0.0384 & 0.0308 \\
& Estimated Value & 0.2731 & 0.3378 \\
50 & Bias & -0.1614 & -0.1527 \\
& MSE & 0.0330 & 0.0284 \\
& Estimated Value & 0.2439 & 0.3040 \\
& Bias & -0.1554 & -0.1519
\end{tabular}




\begin{tabular}{llll} 
Sample & Criteria & $\hat{\lambda}_{M M E}$ & $\hat{\lambda}_{M E M L}$ \\
Size (n) & & 0.3247 & 0.4191 \\
& & -0.1518 & -0.1524 \\
100 & Estimated Value & 0.0254 & 0.0248 \\
& Bias & MSE & \\
\hline
\end{tabular}



Fig. 1. Graph of MSE's of different estimator of Parameter of Laplace distribution with parameter $\lambda$ $=0.5$.

Table 2. Estimated value, Bias and MSE of different estimators of parameter $\lambda$ of Laplace distribution when $\lambda=1, \theta=1.5$ and $\mathrm{c}=1$

\begin{tabular}{llll}
\hline Sample & Criteria & $\hat{\lambda}_{M M E}$ & $\hat{\lambda}_{M E M L}$ \\
Size $(\mathrm{n})$ & & 0.8157 & 0.9539 \\
& Estimated Value & -0.4491 & -0.2991 \\
& Bias & 0.2998 & 0.2112 \\
10 & MSE & 0.5439 & 0.5298 \\
& Estimated Value & -0.3835 & -0.3147 \\
& Bias & 0.2113 & 0.1588 \\
20 & MSE & 0.4148 & 0.4448 \\
& Estimated Value & -0.3429 & -0.3109 \\
& Bias & 0.1567 & 0.1265 \\
30 & MSE & 0.6325 & 0.7145 \\
& Estimated Value & -0.3257 & -0.3041 \\
50 & Bias & 0.1349 & 0.1135 \\
& MSE & 0.7221 & 0.7148 \\
& Estimated Value & -0.3148 & -0.3058 \\
100 & Bias & 0.1164 & 0.1056 \\
& MSE & 0.7161 & 0.7077 \\
& Estimated Value & -0.3038 & -0.3054 \\
\hline
\end{tabular}






Fig. 2. Graph of MSE's of different estimator of Parameter of Laplace distribution with parameter $\lambda$ $=1$.

Table 3. Estimated value, Bias and MSE of different estimators of parameter $\lambda$ of Laplace distribution when $\lambda=1.5, \theta=2$ and $\mathrm{c}=-0.5$

\begin{tabular}{llll}
\hline Sample & Criteria & $\hat{\lambda}_{M M E}$ & $\hat{\lambda}_{M E M L}$ \\
Size (n) & & 0.3445 & 0.3322 \\
\hline 5 & Estimated Value & -0.6818 & -0.2817 \\
& Bias & 0.6820 & 0.4718 \\
10 & MSE & 0.8159 & 0.8587 \\
& Estimated Value & -0.5752 & -0.3890 \\
& Bias & 0.4755 & 0.3084 \\
20 & MSE & 0.6222 & 0.6932 \\
& Estimated Value & -0.5144 & -0.4263 \\
& Bias & 0.3525 & 0.2541 \\
30 & MSE & 1.0115 & 1.0279 \\
& Estimated Value & -0.4913 & -0.4306 \\
50 & Bias & 0.3049 & 0.2358 \\
& MSE & 1.0831 & 1.0885 \\
& Estimated Value & -0.4722 & -0.4418 \\
100 & Bias & 0.2619 & 0.2242 \\
& MSE & 1.0742 & 1.0695 \\
& Estimated Value & -0.4557 & -0.4502 \\
\hline
\end{tabular}




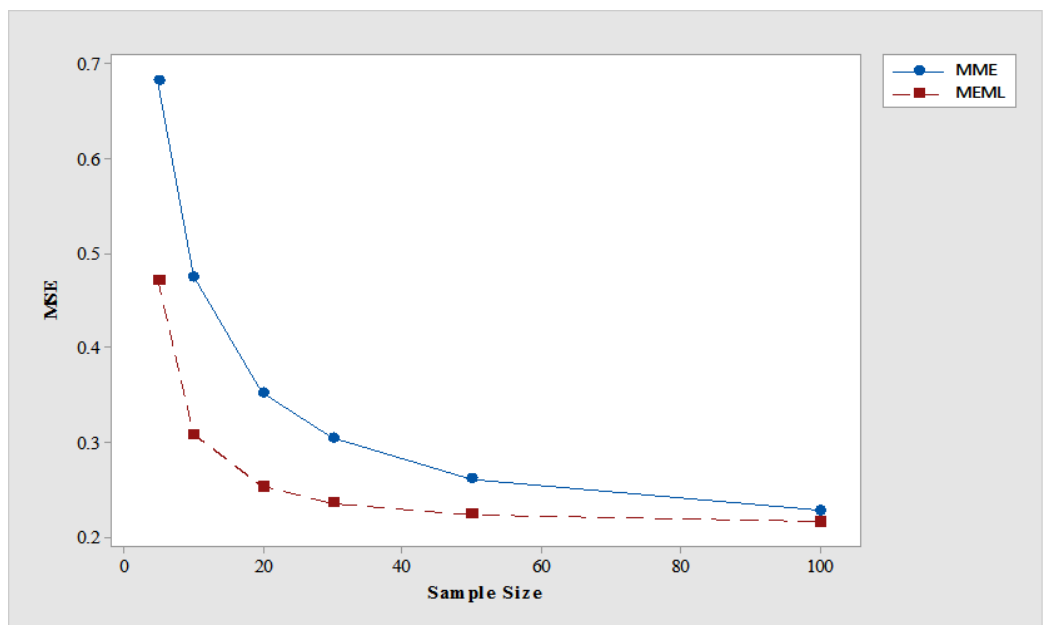

Fig. 3. Graph of MSE's of different estimator of Parameter of Laplace distribution with parameter $\lambda$ $=1.5$.

Table 4. Estimated value, Bias and MSE of different estimators of parameter $\lambda$ of Laplace distribution when $\lambda=2, \theta=2.5$ and $\mathrm{c}=-1$

\begin{tabular}{llll}
\hline $\begin{array}{l}\text { Sample } \\
\text { Size }(\mathrm{n})\end{array}$ & Criteria & $\hat{\lambda}_{M M E}$ & $\hat{\lambda}_{M E M L}$ \\
\hline 5 & Estimated Value & 0.4593 & 0.4714 \\
& Bias & -0.9091 & -0.2711 \\
& MSE & 1.2125 & 0.8407 \\
10 & Estimated Value & 1.0878 & 1.1772 \\
& Bias & -0.7669 & -0.4770 \\
& MSE & 0.8454 & 0.5226 \\
& Estimated Value & 0.8296 & 0.9365 \\
& Bias & -0.6858 & -0.5494 \\
30 & MSE & 0.6266 & 0.4339 \\
& Estimated Value & 0.9802 & 1.2666 \\
& Bias & -0.6541 & -0.5699 \\
50 & MSE & 0.5404 & 0.4111 \\
& Estimated Value & 1.4441 & 1.4587 \\
& Bias & -0.6296 & -0.5832 \\
100 & MSE & 0.4655 & 0.3906 \\
& Estimated Value & 1.4322 & 1.4296 \\
& Bias & -0.6076 & -0.5968 \\
\hline
\end{tabular}




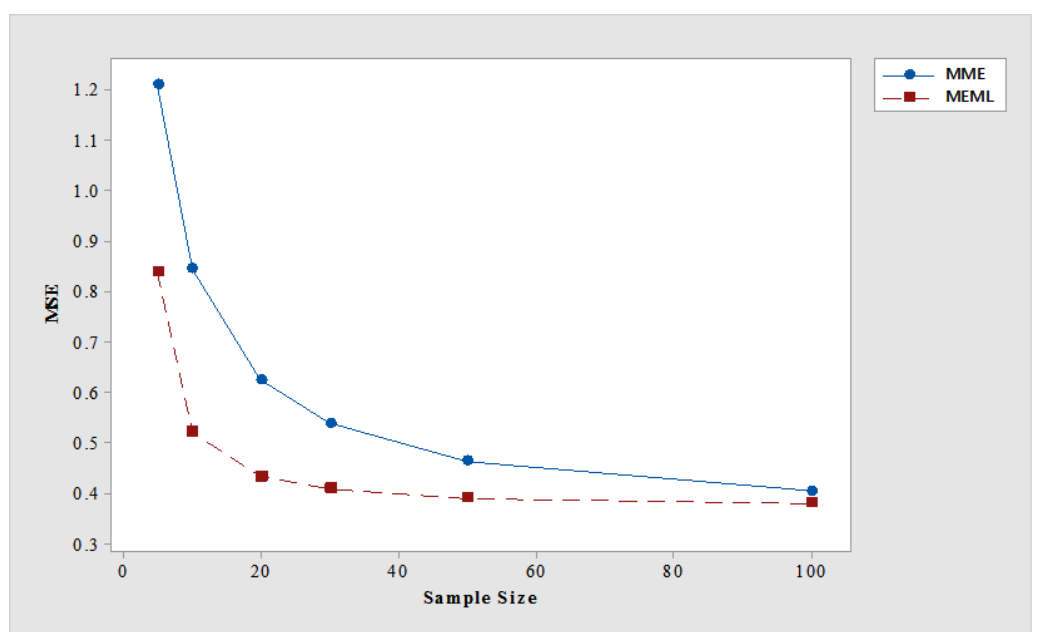

Fig. 4. Graph of MSE's of different estimator of Parameter of Laplace distribution with parameter $\lambda$ $=2$.

\section{Discussion}

The results of the simulation study for estimating the scale parameter $\lambda$ of Laplace distribution when the location parameter $\theta$ is known, are summarized and tabulated in above Tables (1-4) which contains the estimated value, bias and MSE's we have observed. The performance of Bayes estimator under MLINEX loss function with Jeffery prior information is the best estimator, comparing to MM estimator for all sample sizes and with all values of the scale parameter $\lambda$. It is observed that MSE's of all estimators of scale parameter is increasing with the increase of the scale parameter value. Finally, for all parameter values, an obvious reduction in MSE's is observed with the increase in sample size, which matches with the results of Rasheed et al. [3].

\section{Conclusion}

From the above analysis and graphical presentation, it has indicated that minimax estimator under MLINEX loss function is better than moment estimator in the study. Finally concluded that non-classical estimator (minimax estimator under MLINEX loss function) is better than classical estimator (MM estimator).

\section{Acknowledgments}

The researcher wishes to express his gratitude to all the students of various public and private Universities in Bangladesh, which will encourage them to enter into the research arena. The author also expresses special thanks to his wife, for cordial cooperation to make the paper published. 


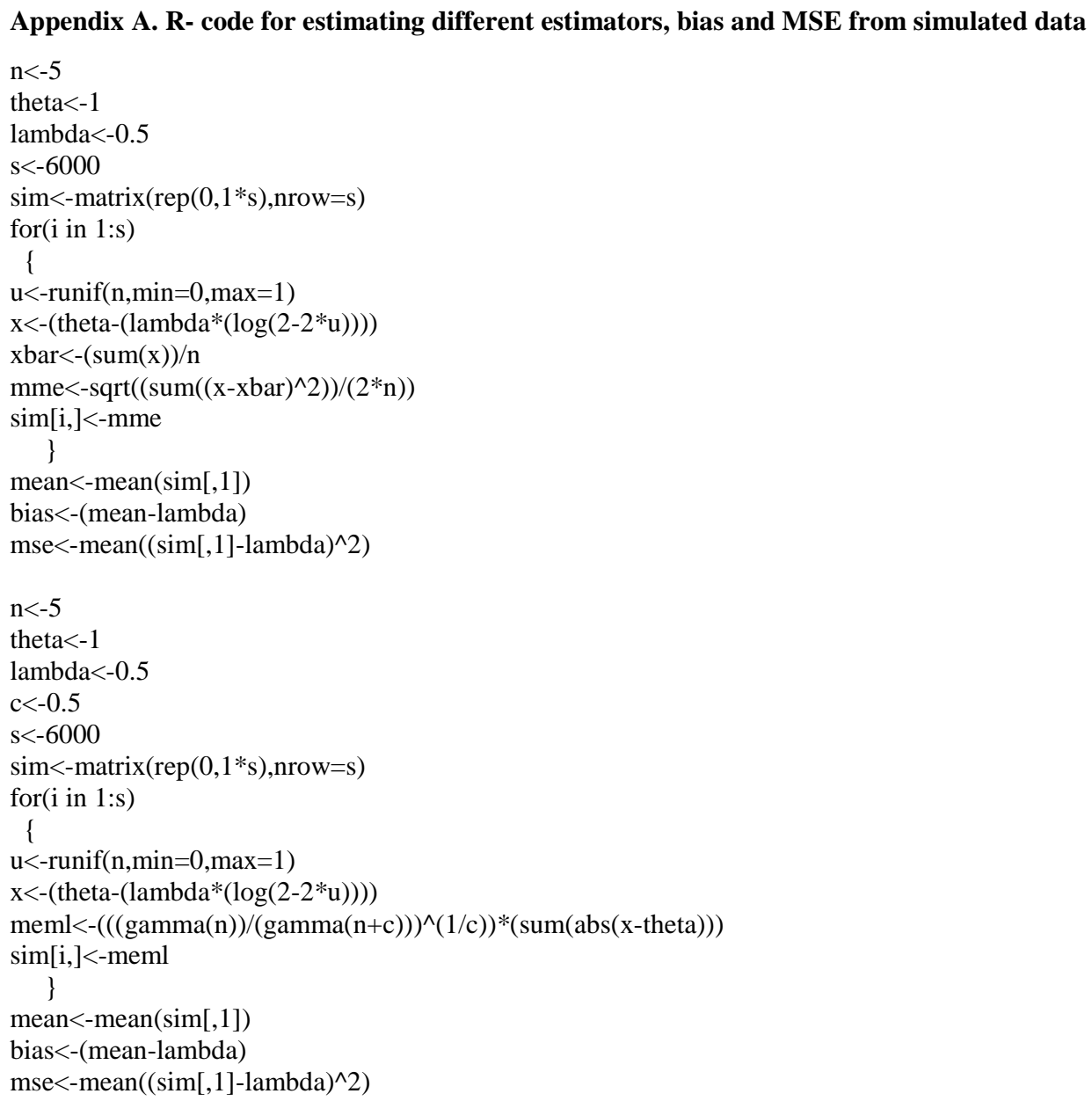

\section{References}

1. A. Wald, Statistical Decision Theory (Mc Graw- Hill, New York, 1950).

2. W. A. J. Al- taie, https://www.researchgate.net/publication/272367648

3. H. A. Rasheed and E. F. Al- Shareefi, Math. Theory Modeling 5, 183 (2015).

4. L. Li, Am. J. Theoretical Appl. Stat. 5, 202 (2016).

5. L. Li, Int. J. Data Sci. Anl. 3, 85(2017).

6. M. R. Hasan and A. R. Baizid, J. Sci. Res. 9, 67 (2017). http://dx.doi.org/10.3329/jsr.v9i1.29308

7. J. Mahanta and M. B. A. Talukdar, J. Sci. Res. 11, 23 (2019). http://dx.doi.org/10.3329/jsr.v11i1.37065

8. https://en.wikipedia.org/wiki/Laplace_distribution\#Probability_density_function (accessed on Dec. 20, 2018).

9. https://en.wikipedia.org/wiki/Laplace_distribution\#Applications (accessed on Dec. 20, 2018).

10. S. A. Nasir and N. J. AL- Anber, J. Math. Stat. 8, 42 (2012).

11. H. S. Al- Kutubi and N. A. Ibrahim, Mal. J. Math. Sci. 3, 297 (2009). 
12. S. Kotz, T. Kozubowski, and K. Podgorski, The Laplace Distribution, and Generalizations: A Revisit with Applications to Communications, Economics, Engineering and Finance, (Springer, Birkhauser Boston, 2001).

13. https://www.youtube.com/watch?v=J-JQ06Racz4 (accessed on Dec. 20, 2018).

14. L. Li, Res. J. Math. Stat. 5, 24 (2013).

15. S. Dey, Data Sci. J. 7, 23 (2008). https://doi.org/10.2481/dsj.7.23

16. A. M. Mood, F. A. Graybill, and D. C. Boes, Introduction to the Theory of Statistics, $3^{\text {rd }}$ Edition (Mc Graw- Hill Book Company, Singapore, 1974) pp. 350. 